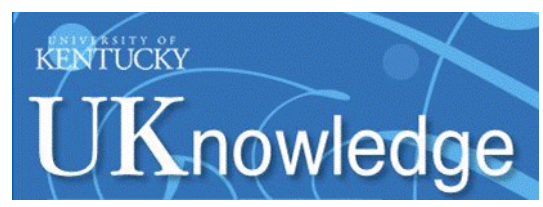

University of Kentucky

UKnowledge

\title{
The Central Molecular Gas Structure in LINERs with Low- Luminosity Active Galactic Nuclei: Evidence for Gradual Disappearance of the Torus
}

\author{
F. Müller-Sánchez \\ Instituto de Astrofísica de Canarias, Spain \\ M. A. Prieto \\ Instituto de Astrofísica de Canarias, Spain \\ M. Mezcua \\ Instituto de Astrofísica de Canarias, Spain \\ R. I. Davies \\ Max Planck Institut für Extraterrestrische Physik, Germany \\ M. A. Malkan \\ University of California - Los Angeles
}

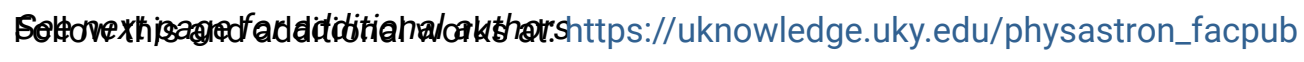

Part of the Astrophysics and Astronomy Commons, and the Physics Commons

Right click to open a feedback form in a new tab to let us know how this document benefits you.

\section{Repository Citation}

Müller-Sánchez, F.; Prieto, M. A.; Mezcua, M.; Davies, R. I.; Malkan, M. A.; and Elitzur, Moshe, "The Central Molecular Gas Structure in LINERs with Low-Luminosity Active Galactic Nuclei: Evidence for Gradual Disappearance of the Torus" (2012). Physics and Astronomy Faculty Publications. 187.

https://uknowledge.uky.edu/physastron_facpub/187

This Article is brought to you for free and open access by the Physics and Astronomy at UKnowledge. It has been accepted for inclusion in Physics and Astronomy Faculty Publications by an authorized administrator of UKnowledge. For more information, please contact UKnowledge@lsv.uky.edu. 


\section{The Central Molecular Gas Structure in LINERs with Low-Luminosity Active Galactic Nuclei: Evidence for Gradual Disappearance of the Torus}

Digital Object Identifier (DOI)

https://doi.org/10.1088/2041-8205/763/1/L1

Notes/Citation Information

Published in The Astrophysical Journal Letters, v. 763, no. 1, L1, p. 1-6.

(c) 2013. The American Astronomical Society. All rights reserved. Printed in the U.S.A.

The copyright holder has granted permission for posting the article here.

Authors

F. Müller-Sánchez, M. A. Prieto, M. Mezcua, R. I. Davies, M. A. Malkan, and Moshe Elitzur 


\title{
THE CENTRAL MOLECULAR GAS STRUCTURE IN LINERS WITH LOW-LUMINOSITY ACTIVE GALACTIC NUCLEI: EVIDENCE FOR GRADUAL DISAPPEARANCE OF THE TORUS*
}

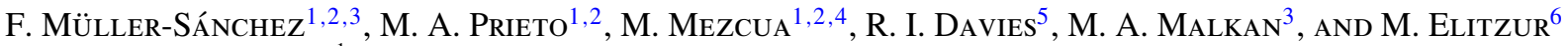 \\ ${ }^{1}$ Instituto de Astrofísica de Canarias, Vía Láctea s/n, La Laguna E-38205, Spain \\ ${ }^{2}$ Departamento de Astrofísica, Facultad de Física, Universidad de la Laguna, Astrofísico Fco. Sánchez s/n, La Laguna E-38207, Spain \\ ${ }^{3}$ Department of Physics and Astronomy, University of California, Los Angeles, CA 90095-1547, USA \\ ${ }^{4}$ Max Planck Institut für Radioastronomie, Auf dem Huegel 69, D-53121 Bonn, Germany \\ ${ }_{6}^{5}$ Max Planck Institut für Extraterrestrische Physik, Postfach 1312, D-85741 Garching, Germany \\ ${ }^{6}$ Department of Physics \& Astronomy, University of Kentucky Lexington, KY 40506-0055, USA \\ Received 2012 September 21; accepted 2012 November 30; published 2012 December 21
}

\begin{abstract}
We present observations of the molecular gas in the nuclear environment of three prototypical low-luminosity active galactic nuclei (LLAGNs), based on VLT/SINFONI AO-assisted integral-field spectroscopy of $\mathrm{H}_{2} 1-0 \mathrm{~S}(1)$ emission at angular resolutions of $\sim 0$ '! 17. On scales of 50-150 pc, the spatial distribution and kinematics of the molecular gas are consistent with a rotating thin disk, where the ratio of rotation $(V)$ to dispersion $(\sigma)$ exceeds unity. However, in the central $50 \mathrm{pc}$, the observations reveal a geometrically and optically thick structure of molecular gas $\left(V / \sigma<1\right.$ and $\left.N_{\mathrm{H}}>10^{23} \mathrm{~cm}^{-2}\right)$ that is likely to be associated with the outer extent of any smaller scale obscuring structure. In contrast to Seyfert galaxies, the molecular gas in LLAGNs has a $V / \sigma<1$ over an area that is $\sim 9$ times smaller and column densities that are on average $\sim 3$ times smaller. We interpret these results as evidence for a gradual disappearance of the nuclear obscuring structure. While a disk wind may not be able to maintain a thick rotating structure at these luminosities, inflow of material into the nuclear region could provide sufficient energy to sustain it. In this context, LLAGNs may represent the final phase of accretion in current theories of torus evolution. While the inflow rate is considerable during the Seyfert phase, it is slowly decreasing, and the collisional disk is gradually transitioning to become geometrically thin. Furthermore, the nuclear region of these LLAGNs is dominated by intermediate-age/old stellar populations (with little or no ongoing star formation), consistent with a late stage of evolution.
\end{abstract}

Key words: galaxies: active - galaxies: kinematics and dynamics - galaxies: nuclei - infrared: galaxies

Online-only material: color figures

\section{INTRODUCTION}

For three decades, the "unified model" of active galactic nuclei (AGNs) has dominated discussion. Its most popular (and extreme) version assumes that AGN types are distinguished only by viewing angle, with the broad lines in Type-2s blocked from our line of sight (LOS) by a highly inclined molecular/ dusty torus. This premise is supposed to hold for all luminosities, from low-luminosity AGNs (LLAGNs) to powerful quasars (Antonucci 1993). More realistic formulations of the torus model involve additional variables such as covering factor or AGN luminosity (see the discussion in Elitzur 2012). Direct evidence for this model comes from spatially resolved interferometric observations of thermal dust in a few prototypical AGNs (e.g., Jaffe et al. 2004; Tristram et al. 2007). However, these observations do not provide sufficient information on the actual geometry (e.g., position angle (P.A.) coverage necessary to constrain shape/orientation) and physical properties (e.g., dynamics) of the obscuring medium.

By means of adaptive optics (AO) integral-field spectroscopy, Hicks et al. (2009, hereafter H09) showed that the molecular gas in the central tens of parsecs of Seyfert galaxies relates directly to the largest structures associated with the obscuring torus, as predicted by clumpy torus models (e.g., Nenkova et al. 2002, 2008; Schartmann et al. 2008): it is in a rotating disk-like distribution, has a high velocity dispersion relative to rotation

\footnotetext{
* Based on observations at the European Southern Observatory VLT (082.B-0709 and 084.B-0568).
}

$(V / \sigma<1)$, and is optically thick (see also Müller-Sánchez et al. 2006, 2009). HCN measurements of Seyfert galaxies suggest that the nuclear dense gas also has a large dispersion (Sani et al. 2012). However, it remains unclear how this vertical structure can be supported. Some models suggest that the torus may be an evolving structure driven by accretion processes operating on scales of tens of parsecs, such as nuclear starbursts or disk instabilities (e.g., Vollmer et al. 2008; Wada et al. 2009; Hopkins et al. 2012). When the inflow rate is low, the thickness and opacity of the torus decrease. Alternatively, by considering both the broad-line region and the torus to be different aspects of a disk wind, Elitzur \& Ho (2009) argue that the torus thickness depends on AGN accretion. They predict that below a given luminosity threshold, a wind with a significant column density $\left(N_{\mathrm{H}}\right)$ can no longer be sustained. In either case, the geometrically and optically thick structure of molecular gas in LLAGNs is expected to disappear.

Observationally, there is an ongoing debate on the existence of an obscuring structure in LLAGNs. ${ }^{7}$ The non-detection of an IR bump in the spectral energy distributions (SEDs) of LLAGNs (Fernández-Ontiveros et al. 2012) provides evidence for unobscured nuclei, as do the compact variable central UV sources observed in both types of LLAGNs (e.g., Maoz et al.

\footnotetext{
7 The bulk of the LLAGN population reside in low-ionization nuclear emission-line regions (LINERs; Ho 2008). Since the bolometric luminosity threshold for separating low- and high-luminosity AGNs is roughly defined as a few $10^{42} \mathrm{erg} \mathrm{s}^{-1}$ (Ho 2009), some LLAGNs reside in Seyfert galaxies. Strictly speaking, we study LINERs/LLAGNs here, but refer to them as LLAGNs.
} 
Table 1

Summary of Basic Data for LLAGNs and Quantities Derived from the $\mathrm{H}_{2}$ 1-0 S(1) Kinematics

\begin{tabular}{|c|c|c|c|c|c|c|c|c|c|c|c|c|c|c|}
\hline \multicolumn{4}{|c|}{ Basic Data } & \multicolumn{5}{|c|}{ Global Derived Parameters } & \multicolumn{3}{|c|}{ At $r=25 \mathrm{pc}$} & \multicolumn{3}{|c|}{ At $r=100 \mathrm{pc}$} \\
\hline Object & $\begin{array}{l}\text { AGN } \\
\text { Type }^{\mathrm{a}}\end{array}$ & $\begin{array}{c}D^{\mathrm{a}} \\
(\mathrm{Mpc})\end{array}$ & $\begin{array}{l}\log L_{\text {bol }} \mathrm{b} \\
\left(\mathrm{erg} \mathrm{s}^{-1}\right)\end{array}$ & $\begin{array}{l}\text { P.A. } \\
\left({ }^{\circ}\right)\end{array}$ & $\begin{array}{c}i \\
\left(^{\circ}\right)\end{array}$ & $\begin{array}{l}R_{1 / \mathrm{e}} \\
(\mathrm{pc})\end{array}$ & $\begin{array}{c}\log M_{\mathrm{BH}}{ }^{\mathrm{c}} \\
\left(M_{\odot}\right)\end{array}$ & $\log L_{\text {bol }} / L_{\text {Edd }}$ & $V / \sigma$ & $\begin{array}{c}\log M_{\mathrm{dyn}} \mathrm{d} \\
\left(M_{\odot}\right)\end{array}$ & $\begin{array}{c}\log N_{\mathrm{H}} \mathrm{e} \\
\left(\mathrm{cm}^{-2}\right)\end{array}$ & $V / \sigma$ & $\begin{array}{c}\log M_{\text {dyn }}{ }^{\mathrm{f}} \\
\left(M_{\odot}\right)\end{array}$ & $\begin{array}{c}\log N_{\mathrm{H}} \mathrm{e} \\
\left(\mathrm{cm}^{-2}\right)\end{array}$ \\
\hline NGC 1052 & L1.9 & 18 & 41.4 & $30 \pm 5$ & $55 \pm 4$ & $50 \pm 7$ & $8.0_{-0.1}^{+0.2}$ & $-4.9_{-0.1}^{+0.2}$ & $1.0 \pm 0.3$ & $8.3 \pm 0.1$ & $23.2 \pm 0.5$ & $4 \pm 1.0$ & $8.4_{-0.1}^{+0.2}$ & $22.1 \pm 0.5$ \\
\hline NGC 2911 & L2 & 40 & 42.0 & $-156_{-4}^{+7}$ & $57 \pm 5$ & $170 \pm 15$ & $8.2_{-0.2}^{+0.3}$ & $-4.5_{-0.2}^{+0.3}$ & $0.6 \pm 0.2$ & $8.6 \pm 0.2$ & $23.5 \pm 0.5$ & $3 \pm 0.7$ & $9.0_{-0.3}^{+0.2}$ & $22.8 \pm 0.5$ \\
\hline NGC 3169 & L2 & 20 & 42.4 & $47 \pm 3$ & $43 \pm 6$ & $120 \pm 10$ & $7.9_{-0.1}^{+0.2}$ & $-4.0_{-0.1}^{+0.2}$ & $0.9 \pm 0.3$ & $8.2 \pm 0.2$ & $23.1 \pm 0.5$ & $7 \pm 1.5$ & $8.7_{-0.2}^{+0.3}$ & $22.5 \pm 0.5$ \\
\hline NGC 1097 & L1 & 18 & 41.6 & $-52 \pm 5$ & $42 \pm 5$ & $\ldots$ & $8.1_{-0.2}^{+0.2}$ & $-4.4_{-0.2}^{+0.2}$ & $1.2 \pm 0.4$ & $8.2 \pm 0.2$ & $23.0 \pm 0.5$ & $4 \pm 1.2$ & $8.9_{-0.2}^{+0.2}$ & $22.6 \pm 0.5$ \\
\hline
\end{tabular}

Notes. Quoted uncertainties are 1 standard deviation of the fit, except for $N_{\mathrm{H}}$, which is delimited by the assumed gas fraction interval.

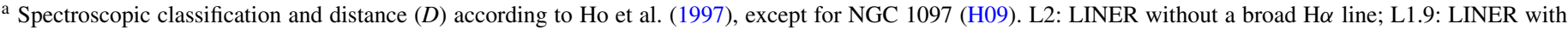

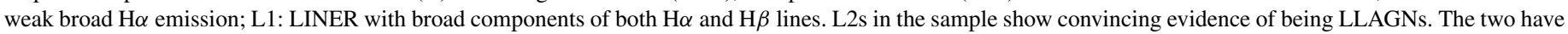
a high brightness-temperature radio core, which is also a hard X-ray point source (Terashima \& Wilson 2003; Wrobel et al. 1984; Ellis \& O'Sullivan 2006).

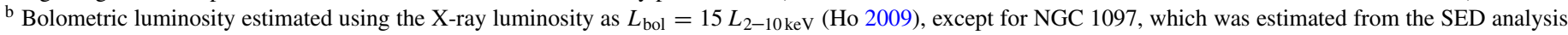
(Prieto et al. 2010). X-ray luminosities taken from Ellis \& O’Sullivan (2006) and Terashima \& Wilson (2003).

c Black hole mass derived from the dynamical models, except for NGC 1097 (Davies et al. 2007).

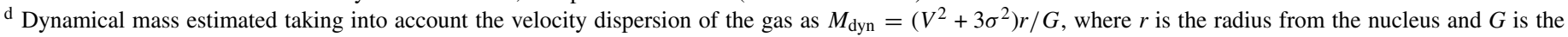
gravitational constant.

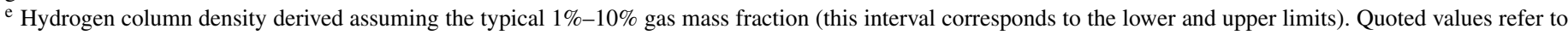
$3.2 \%$ gas fraction (see the text for details).

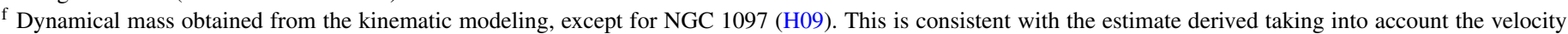
dispersion at this radius within the uncertainties.

2005). Furthermore, X-ray studies of nearby galaxies have found a correlation between $N_{\mathrm{H}}$ and X-ray luminosity (e.g., Zhang et al. 2009).

However, some LLAGNs do exhibit high $N_{\mathrm{H}}$ (e.g., NGC 3169; Terashima \& Wilson 2003). The detection of polarized broad lines and ionization bicones in some LLAGNs provides additional support for the presence of an obscuring torus (Barth et al. 1999; Pogge et al. 2000).

In an effort to characterize for the first time the properties of the molecular gas in the central regions of LLAGNs, we have observed three prototypical LINERs/LLAGNs (Table 1) using AO-assisted $K$-band integral-field spectroscopy. The $2.12 \mu \mathrm{m}$ $\mathrm{H}_{2}$ 1-0 S(1) emission line is used to analyze the two-dimensional (2D) distribution and kinematics of the gas within the central $150 \mathrm{pc}$. These techniques have already been applied to study the properties of $\mathrm{H}_{2}$ in NGC 1097 (H09; Davies et al. 2009). Here we bring those data together with new data on three additional objects (Sections 2 and 3) to probe the putative obscuring medium in LLAGNs.

\section{OBSERVATIONS AND DATA PROCESSING}

The galaxies analyzed here were observed with AO and SINFONI at the Very Large Telescope (VLT) in two separate runs between 2009 March and 2010 March. SINFONI provides spectra for every pixel in a contiguous 2D field with $64 \times$ 32 pixels (Eisenhauer et al. 2003). The pixel scale in all observations was $00^{\prime} .05 \times 0 . ' 1$, resulting in a field of view of 3 .'2 $2 \times 3 . .2$. The spectral range was similar for all runs $(1.95-2.45 \mu \mathrm{m})$ with typical spectral resolution $R \sim 4000$ (FWHM $\sim 70 \mathrm{~km} \mathrm{~s}^{-1}$ ). For each galaxy, the nucleus was used as Natural Guide Star (NGS) by the AO module. The exposure times for NGC 1052, NGC 2911, and NGC 3169 were 180, 20, and 60 minutes, respectively. The FWHM angular resolution, measured from the spatially unresolved non-stellar continuum in $K$ band, is $\sim 0$ '. 17 in NGC 1052 and NGC 3169 , corresponding to 15 and 16 pc, respectively, and $\sim 0.23$ (45 pc) in NGC 2911. Data reduction was performed using the SINFONI data reduction package SPRED (Abuter et al. 2005). Nearby standard stars (A- or B-type) were observed close in time to the science frames

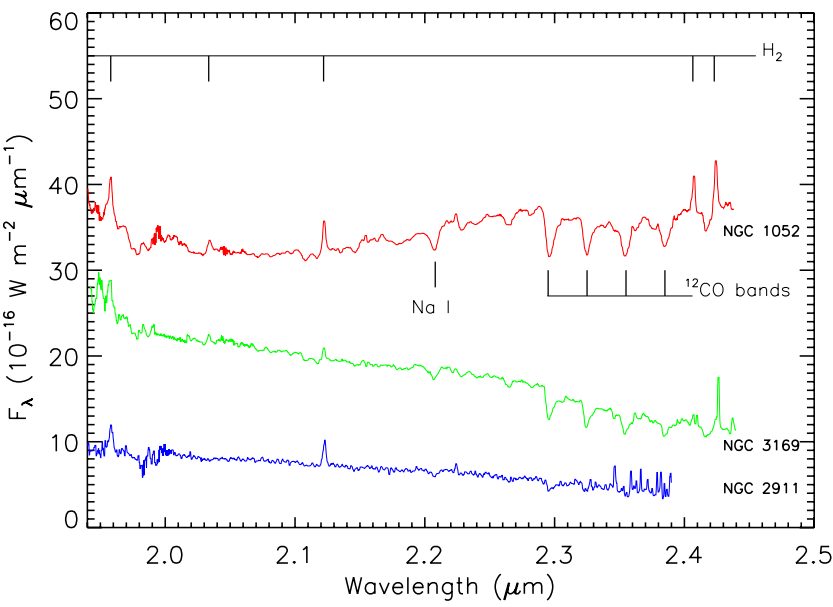

Figure 1. $K$-band nuclear spectra of LLAGNs extracted from our SINFONI data in $r=0^{\prime \prime} 3$ apertures. The prominent emission lines are from $\mathrm{H}_{2}$ (marked at the top of the figure), the strongest being $1-0 \mathrm{~S}(1)$ at $2.12 \mu \mathrm{m}$. Several stellar absorption features (like the ${ }^{12} \mathrm{CO}$ band heads or $\mathrm{NaI}$ ), characteristic of evolved stellar populations, are also noticeable. All $K$-band ionization lines (including $\mathrm{Br} \gamma$ ) are absent in these objects. NGC 1097 exhibits a similar spectrum, but $\mathrm{Br} \gamma$ is marginally detected (Davies et al. 2007). Since $\mathrm{Br} \gamma$ is normally considered a tracer of young stars, the marginally/non-detection of this line indicates little (as the very young and compact starburst in NGC 1097; Davies et al. 2007) or no ongoing star formation.

(A color version of this figure is available in the online journal.)

and used for telluric correction and flux calibration. In addition, flux calibration was cross-checked with VLT/NACO and Two Micron All Sky Survey photometric data in $1^{\prime \prime}-4^{\prime \prime}$ apertures.

The resulting spectra show several emission lines of $\mathrm{H}_{2}$ and stellar absorption features (Figure 1). The 2D properties of $\mathrm{H}_{2}$ 1-0 S(1) were extracted using the code LINEFIT (Davies et al. 2009). This procedure corrects automatically for instrumental broadening. Typical uncertainties in the kinematic measurements are between 5 and $20 \mathrm{~km} \mathrm{~s}^{-1}$.

\section{RESULTS AND ANALYSIS}

The SINFONI data reveal the morphology and kinematics of the molecular gas in unprecedented detail (Figure 2; see H09 

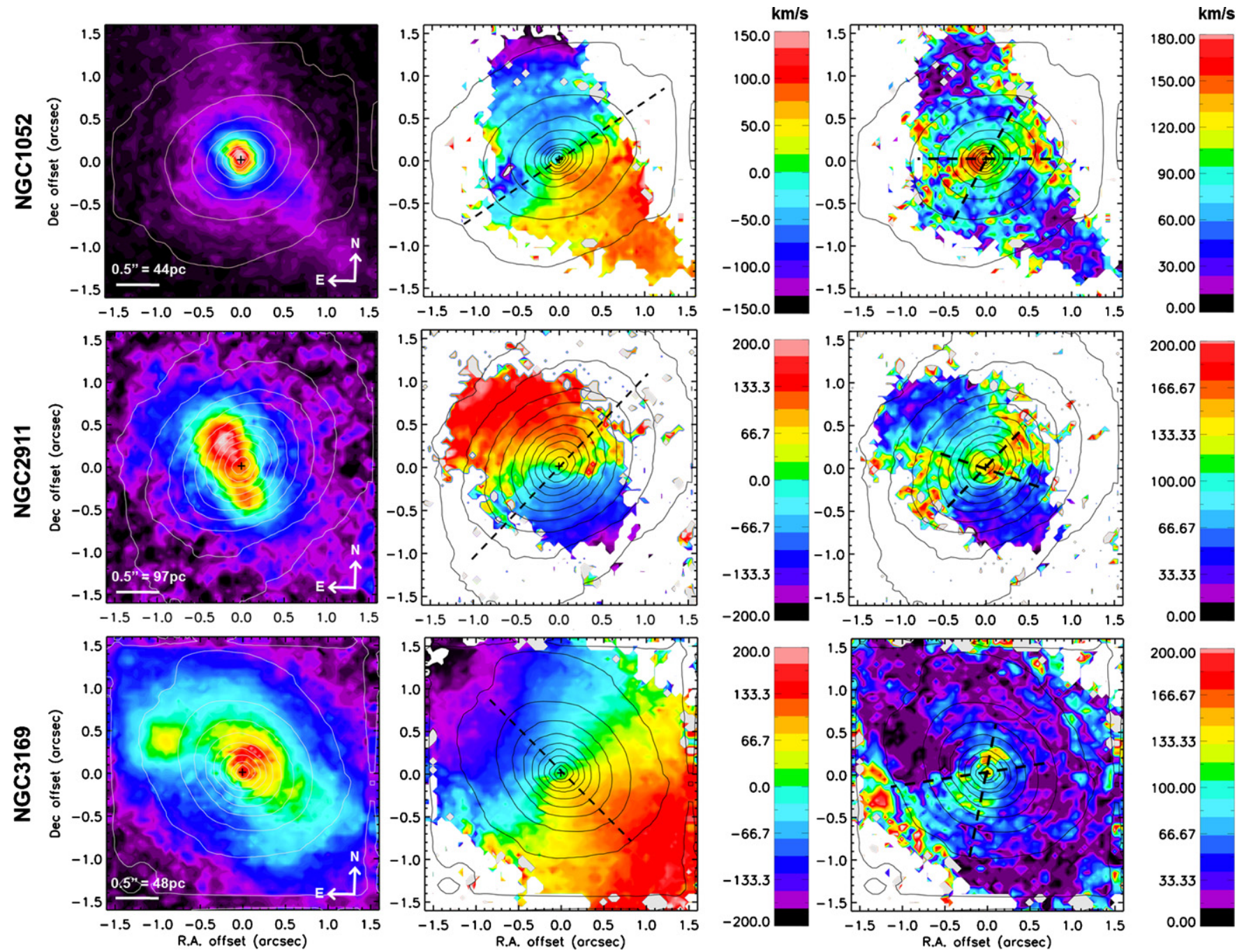

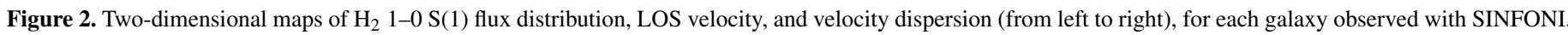

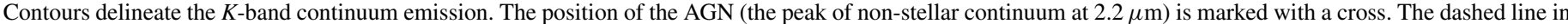

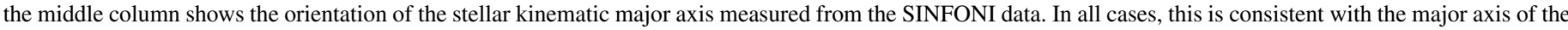

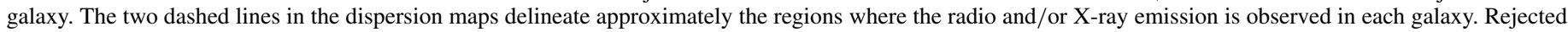
pixels in the velocity and dispersion maps are those with a flux density lower than $5 \%$ of the peak of $\mathrm{H}_{2}$ emission.

(A color version of this figure is available in the online journal.)

for the maps of NGC 1097). Similar morphologies are observed in the three sample galaxies. The flux distributions show bright central structures with elongations from northeast to southwest and morphologies suggesting filamentary formations. While in NGC 3169 (morphological type S), the photometric P.A. of $\mathrm{H}_{2}$ is similar to the P.A. of the stellar continuum, in NGC 1052 and NGC 2911 (type E4 and S0, respectively), the P.A. of $\mathrm{H}_{2}$ is strongly misaligned (by $\sim 70^{\circ}-80^{\circ}$ ) with that of the stars and the galactic major axis, indicative of an external origin of the gas, probably being acquired through merging, as has been proposed for elliptical galaxies (Morganti et al. 2006).

The velocity fields of $\mathrm{H}_{2}$ exhibit azimuthal symmetry, a zerovelocity axis (or kinematic minor axis) almost perpendicular to the kinematic major axis (which is also the photometric major axis in all cases), and small twists of the isovelocity contours. Taken together, these features provide strong qualitative evidence for rotation in a disk, but with the presence of radial flows in the nuclear region. In NGC 1052 and NGC 2911, the gas has a kinematic major axis that is not aligned with the stellar rotation axis (Figure 2), providing strong support to the hypothesis of an external origin of the molecular gas.
While the $\mathrm{H}_{2}$ velocity fields indicate rotating disks, the dispersion maps suggest that these disks are fairly thin in the outer $r>0$ '. $4\left(\sigma \sim 20-30 \mathrm{~km} \mathrm{~s}^{-1}\right)$, but remarkably thick in the vicinity of the AGN ( $\sigma$ increases up to $\sim 150 \mathrm{~km} \mathrm{~s}^{-1}$ ). In all cases, $\sigma$ also increases in a direction almost perpendicular to the $\mathrm{H}_{2}$ kinematic major axis (in NGC 3169 , this is observed only in the southeast; Figure 2). Our experience with previous integralfield observations of other galaxies reveals that increases in dispersion in particular directions surrounding the AGN are usually related to the presence of a passing radio jet and/or outflows (Müller-Sánchez et al. 2011). Radio and X-ray observations of NGC 1052 and NGC 3169 reveal extended emission in the direction of the enhancements in $\sigma$ (Kadler et al. 2004; Hummel et al. 1987; Terashima \& Wilson 2003). A similar situation is observed in radio maps of NGC 2911 (Wrobel et al. 1984; Condon et al. 1991). Thus, the regions of enhanced $\sigma$-values (delineated by dashed lines in the dispersion maps, Figure 2) are probably due to radio jets or AGN-driven outflows.

For quantitative modeling, we adopted an axisymmetric rotating disk generated by the code DYSMAL (as in NGC 1097; Davies et al. 2009, 2011). For determining the disk parameters 
and their uncertainties, we adopted an exponential mass distribution of outer radius $1^{\prime \prime}$. 6 . We then varied inclination, P.A., total mass, black hole $(\mathrm{BH})$ mass, and radial scale length $\left(R_{1 / \mathrm{e}}\right)$ to minimize $\chi^{2}$ in the residual velocity and dispersion maps. The regions perpendicular to the kinematic major axis containing high $\sigma$-values (the area inside the dashed lines in the dispersion maps, Figure 2), which are likely of non-gravitational nature, were not included in the fit. The smearing due to the instrumental resolution is taken into account by convolving the inclined model with the Gaussian point-spread function of the appropriate width. Input errors in the $\chi^{2}$ evaluation were the $1 \sigma$ fit uncertainties of the line profiles. All uncertainties given for the derived parameters are $68 \%$ confidence.

The best-fitting models are presented in Table 1 and illustrated with one-dimensional cuts along the kinematic major axis (Figure 3). The models provide a good fit to the observed LOS velocity and $\sigma$ curves, but the relatively large nuclear dispersion (at $r<0$.'4) cannot be accounted for by the models. The dominant kinematic component in all cases is well described by circular motion. However, the velocity residual maps of NGC 1052 and NGC 2911 show non-circular velocities of up to $\sim 60 \mathrm{~km} \mathrm{~s}^{-1}$ within $1^{\prime \prime} .5$ of the nucleus. Since the purpose of this investigation is to derive the best-fitting velocity field and study the bulk properties of $\mathrm{H}_{2}$, we do not discuss the residuals here (including the regions where the outflows are observed). A detailed analysis of the kinematics in these two galaxies will be presented in another publication. Overall, the observed properties of the nuclear $\mathrm{H}_{2}$ in these LLAGNs indicate a disklike distribution with significant rotation and high velocity dispersion in the central $r<0$ '. 4 .

\section{DISCUSSION}

On scales of $r>50 \mathrm{pc}(\sim 100 \mathrm{pc}$ in NGC 2911), the properties of the molecular gas are consistent with a thin-disk model (Figure 3). Inside this radius, the dispersion increases faster than the models, and within $r<25$ pc ( $\sim 50$ pc in NGC 2911, but we use $25 \mathrm{pc}$ for comparison purposes), the ratio $V / \sigma$ is $\leqslant 1$, implying that the gas on these scales must be supported by rotation and random motions within a spheroidal structure (e.g., a geometrically thick disk). In addition, the gas in this region is optically thick. From the estimated dynamical masses, and assuming a typical gas fraction $\left(f_{g}\right)$ in the centers of galaxies between $1 \%$ and $10 \%{ }^{8}$ (H09; Hopkins et al. 2012), we obtain an average $N_{\mathrm{H}}$ of $1.7 \times 10^{23} \mathrm{~cm}^{-2}$ (Table 1). In NGC 1052 and NGC 3169, the estimated $N_{\mathrm{H}}$ is consistent with that derived from X-ray spectroscopy (Terashima \& Wilson 2003; Kadler et al. 2004). Taken together, these characteristics suggest a rotating, geometrically and optically thick disk, and are consistent with those expected for the molecular torus in unification models. A similar conclusion was reached for the nuclear $\mathrm{H}_{2}$ in NGC 1097 (H09). Therefore, we refer to this structure as "large-scale torus," keeping in mind that the obscuring material probed by mid-IR interferometry of Seyferts has a size of a few pc or less (e.g., Jaffe et al. 2004), and that this is likely responsible for other functions such as collimation of ionization cones (Müller-Sánchez et al. 2011). Nevertheless, the contribution of the large-scale torus to AGN obscuration may be considerable, as suggested by current clumpy torus models (e.g., Schartmann et al. 2008).

\footnotetext{
8 The gas mass can also be estimated from the luminosity of 1-0 S(1) using an appropriate conversion factor (Müller-Sánchez et al. 2006). We used the same factor as $\mathrm{H} 09$ of $430 M_{\odot} / L_{\odot}$ (which gives $f_{g} \sim 10 \%$ for Seyferts), and derived an average $f_{g} \sim 3.2 \%$ in the central $50 \mathrm{pc}$ of our sample of LLAGNs. This is the value used throughout the paper. CO measurements of LLAGNs also suggest $f_{g}$ of a few percent (Raluy et al. 1998; Tan et al. 2008).
}

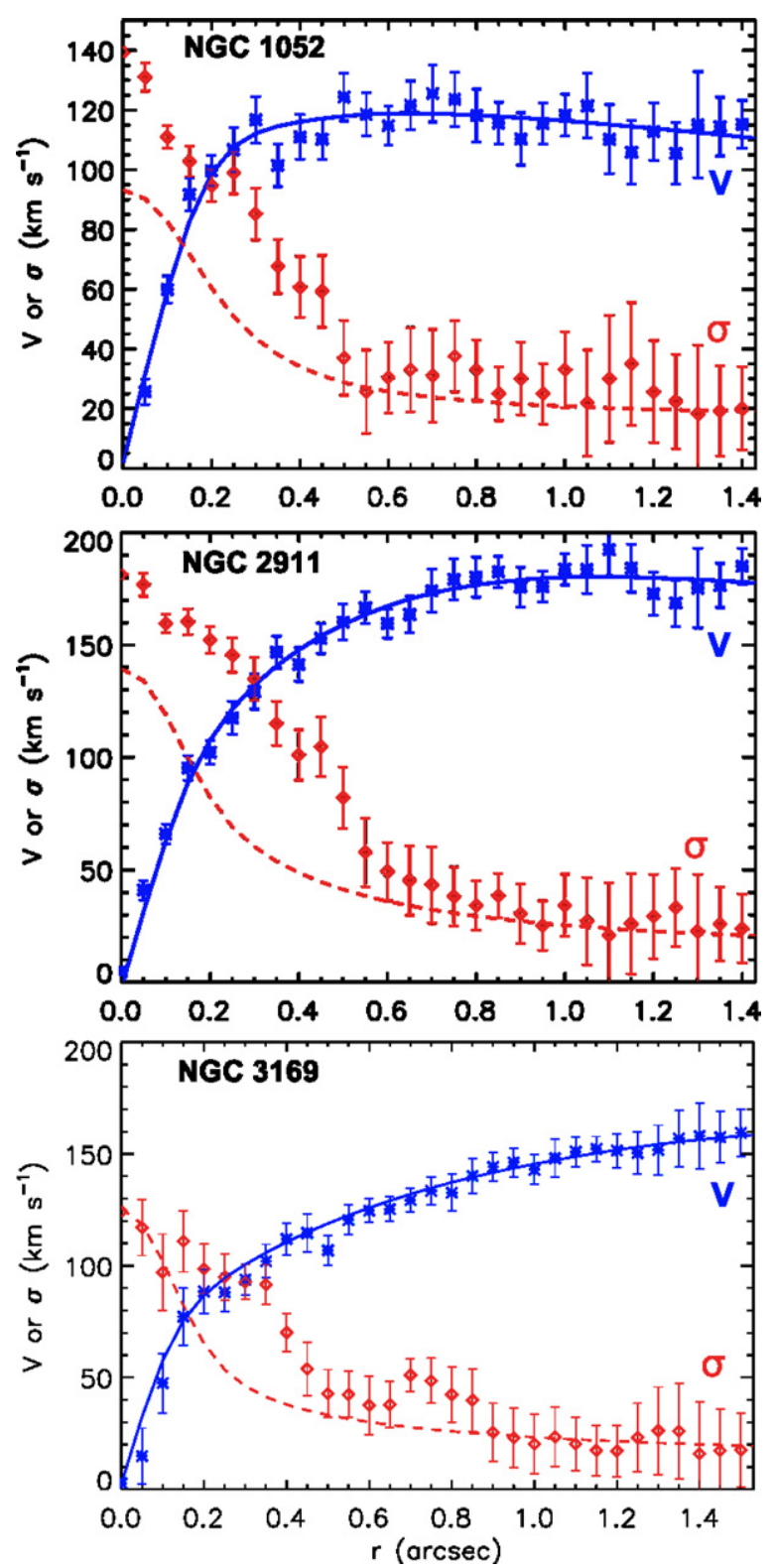

Figure 3. Velocity and dispersion distributions of the molecular gas along the $\mathrm{H}_{2}$ kinematic major axis of the sample galaxies (see $\mathrm{H} 09$ for the curves of NGC 1097). Asterisks represent LOS-velocity measurements and open diamonds dispersion values. Continuous curves denote the best-fitting exponential disk model to the velocity data points, and dashed curves the same models but for $\sigma$. In all panels, vertical error bars represent $1 \sigma$ uncertainties in the measurements.

(A color version of this figure is available in the online journal.)

The large-scale torus in LLAGNs appears to be different from the one in Seyfert galaxies. This conclusion is apparent from the ratio of azimuthally averaged $V / \sigma$ as function of radius (Figure 4). For comparison purposes, we also plotted the average of this function for six Seyfert and two starburst galaxies observed with SINFONI on the same spatial scales $(r \sim 150 \mathrm{pc})$. On average, for LLAGNs, $V / \sigma$ is low $(0.8 \pm 0.3)$ at $r=25 \mathrm{pc}$ and high $(4.6 \pm 1.2)$ at $100 \mathrm{pc}$. Thus, random motions are significant within $r<25 \mathrm{pc}$, despite the rotation of the gas. In contrast, starburst galaxies exhibit a high $V / \sigma$ at any radius consistent with a thin disk (an almost uniform dispersion map with values $<30 \mathrm{~km} \mathrm{~s}^{-1}$, i.e., no significant thickening at the center), and Seyfert galaxies show low $V / \sigma$ at radii up to $100 \mathrm{pc}$, suggesting that the geometrically thick 


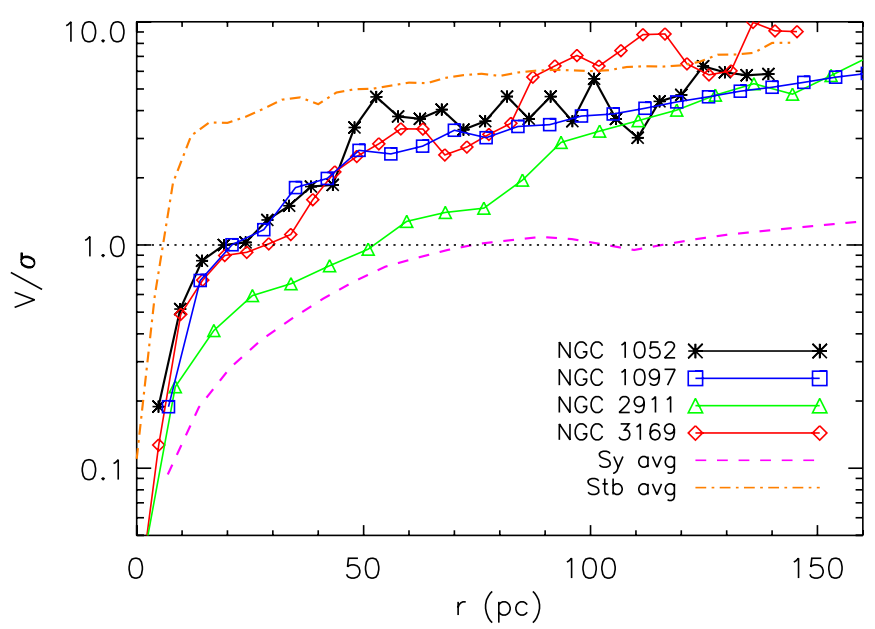

Figure 4. Azimuthally averaged $V / \sigma$ vs. radius for each galaxy as indicated by the legend. The dotted horizontal line shows the point where $V / \sigma=1$. The typical error of the $V / \sigma$ estimates is $35 \%$. The average $V / \sigma$ as a function of radius was obtained for Seyferts from the data of NGC 3783 and NGC 7469 (H09), NGC 2992 (Friedrich et al. 2010), NGC 6814 (Müller-Sánchez et al. 2011), NGC 1566, and NGC 3081 (this work); and for starburst galaxies from NGC 253 and NGC 4945 (this work).

(A color version of this figure is available in the online journal.)

disk in these objects is more extended than in LLAGNs. The obscuration in LLAGNs is probably also lower than in Seyferts. The average 1-0 S(1) luminosity of the LLAGN in our sample within $r=25 \mathrm{pc}$ is $\sim 1.5 \times 10^{4} L_{\odot}$, approximately 3.2 times smaller than the average found for Seyfert galaxies (H09). If the $\mathrm{H}_{2}$ luminosity reflects gas mass (rather than differences in excitation), then the LLAGN in our sample have gas masses (and in consequence $N_{\mathrm{H}}$ ) on average $\sim 3.2$ times smaller than Seyferts (H09). This is consistent with $\mathrm{CO}$ measurements of local AGNs (including NGC 1097 and NGC 1052; Raluy et al. 1998; H09), which indicate that there is more gas in the centers of Seyferts than in LLAGNs.

Figure 4 clearly shows that the molecular gas in LLAGNs is in an intermediate state between an extended large-scale torus (a thick disk) and no large-scale torus at all (a thin disk). We interpret this structure as a large-scale torus in the process of gradual disappearance. This conclusion is supported by the theory of torus evolution proposed by Vollmer et al. (2008). In this model, a torus passes through three different phases dictated by an external mass accretion rate. Initiated by a massive gas infall, a turbulent thin disk is formed in which starbursts occur. Such disks, characterized by the production of several star clusters and high star formation rates (SFR $>10 M_{\odot} \mathrm{yr}^{-1}$ ), are observed in local starburst galaxies (Müller-Sánchez et al. 2010, Figure 4). Once supernovae remove the intercloud medium, a collisional thick disk of dense gas clouds is formed with a decreasing, but still high, accretion rate. The collisional thick disk also forms stars, but with an efficiency of $\sim 10 \%$ when compared to the previous stage (SFR $\sim 1 M_{\odot} \mathrm{yr}^{-1}$ ). The Seyferts in the SINFONI subsample (Figure 4) are interpreted to be in this second phase (H09). The mass inflow rate is slowly decreasing, and the collisional disk is gradually transitioning to become geometrically thin. The SFR reaches a minimum, and the nuclear region is dominated by intermediate-age/old stellar populations. Based on the observed properties, the LLAGN in our sample are in this transitional phase (Figures 1 and 4). When the inflow rate has significantly decreased, the collisional torus either completely disappears (there is very little gas left) or becomes thin and transparent as the circumnuclear disk in the Galactic Center.

The proposed scenario reveals a possible Starburst $\rightarrow$ Seyfert $\rightarrow$ LINER/HII $\rightarrow$ LINER $\rightarrow$ Passive Galaxies evolutionary sequence of AGN activity and $\mathrm{BH}$ growth. The initial phase of activity of a galactic nucleus is characterized by vigorous star formation. After $\sim 10 \mathrm{Myr}$, significant accretion onto the $\mathrm{BH}$ triggers the Seyfert phase. This highly active phase may be modulated by feeding and feedback processes. The final stages of accretion are observed in LINERs, in which massive BHs have already formed. This picture is supported by recent observational studies of local galactic nuclei (e.g., Constantin et al. 2009; Schawinski et al. 2010), and numerical simulations of the origin and evolution of luminous AGNs (e.g., Di Matteo et al. 2005; Hopkins et al. 2006).

The kinematic signatures of outflows detected in the dispersion maps suggest that a disk wind may still be present. In the wind scenario, the main channel for release of energy may be switching at low luminosities from AGN-driven outflow to radio jets. Thus, the regions in which we detect high $\sigma$ could be interpreted as gas from the Interstellar Medium (ISM) shocked by a radio jet. However, it seems unlikely that this mechanism is also responsible for the high dispersion measured in the central $50 \mathrm{pc}$ (at least not in its most part). First, the circular symmetry of these regions (extending out to $r \sim 25-50 \mathrm{pc}$ ) is not consistent with that expected from a collimated jet. In addition, in some objects, there is no clear evidence of a radio jet (e.g., NGC 1097; Orienti \& Prieto 2010), and yet a low $V / \sigma$ is observed (Figure 4). Although a contribution from the disk wind to the large-scale torus seems unlikely, it could significantly contribute to sustain any smaller scale torus (the threshold for an $M_{\mathrm{BH}}=1 \times 10^{8} M_{\odot}$ is $L_{\text {bol }} \gtrsim 2 \times 10^{40} \mathrm{erg} \mathrm{s}^{-1}$; Elitzur $\&$ Ho 2009). The dust sublimation radius in LLAGNs with $L_{\text {bol }}=10^{42} \mathrm{erg} \mathrm{s}^{-1}$ is $r_{\text {sub }}=0.4\left(L_{\text {bol }} / 10^{45}\right)^{1 / 2} \mathrm{pc} \sim 0.012 \mathrm{pc}$ (Nenkova et al. 2008). The outer radius of the "small-scale" torus is then $R=50-100 r_{\text {sub }}=0.6-1.2$ pc (Tristram \& Schartmann 2011), which, at our resolution, is unresolved. These results suggest that the torus comprises at least two components on different scales each of which fulfills different roles. On scales of $\sim 15$ pc and larger, our results reveal a diffuse and clumpy envelope which represents the reservoir of gas necessary to feed the AGN, and possibly contributes to nuclear obscuration. At smaller scales, IR interferometry provides evidence for a compact torus which is likely responsible for most of the obscuration and collimation.

Our integral-field data confirm the presence of an obscuring structure of molecular gas in the central $50 \mathrm{pc}$ of LLAGNs, which indicates that in these objects there is potentially enough material to fuel the AGN. However, it appears to be disappearing as indicated by the lower column densities and sizes compared with those of Seyfert galaxies. Particularly interesting would be a study of the gas kinematics in AGNs having lower luminosities and/or Eddington ratios than the ones presented here. In these objects, either there is no detectable nuclear warm- $\mathrm{H}_{2}$ structure (as in M87 and some other LLAGNs observed with AO; Gebhardt et al. 2011; A. Barth, private communication) or it is thin and transparent as predicted by the torus evolution model (Vollmer et al. 2008).

We thank the staff of ESO for their support, Aaron Barth for helpful discussions, and the anonymous referee for useful comments. We acknowledge financial support from CONACYT and COST Action MP0905 Black Holes in a Violent Universe. 


\section{REFERENCES}

Abuter, R., Schreiber, J., Eisenhauer, F., et al. 2005, NewAR, 50, 398 Antonucci, R. 1993, ARA\&A, 31, 473

Barth, A., Filippenko, A. V., \& Moran, E. C. 1999, ApJ, 525, 673

Condon, J. J., Frayer, D. T., \& Broderick, J. J. 1991, AJ, 101, 362

Constantin, A., Green, P., Aldcroft, T., et al. 2009, ApJ, 705, 1336

Davies, R. I., Förster Schreiber, N. M., Cresci, G., et al. 2011, ApJ, 741, 69

Davies, R. I., Maciejewski, W., Hicks, E. K. S., et al. 2009, ApJ, 702, 114

Davies, R. I., Müller-Sánchez, F., Genzel, R., et al. 2007, ApJ, 671, 1388

Di Matteo, T., Springel, V., \& Hernquist, L. 2005, Natur, 433, 604

Eisenhauer, F., Abuter, R., Bickert, K., et al. 2003, Proc. SPIE, 4841, 1548

Elitzur, M. 2012, ApJL, 747, 33

Elitzur, M., \& Ho, L. C. 2009, ApJL, 701, 91

Ellis, S. C., \& O'Sullivan, E. 2006, MNRAS, 367, 627

Fernández-Ontiveros, J. A., Prieto, M. A., Acosta-Pulido, J. A., \& Montes, M. 2012, JPhCS, 372, 012006

Friedrich, S., Davies, R. I., Hicks, E., et al. 2010, A\&A, 519, 79

Gebhardt, K., Adams, J., Richstone, D., et al. 2011, ApJ, 729, 119

Hicks, E. K. S., Davies, R. I., Malkan, M., et al. 2009, ApJ, 696, 448 (H09)

Ho, L. C. 2008, ARA\&A, 46, 475

Ho, L. C. 2009, ApJ, 699, 626

Ho, L. C., Filippenko, A. V., \& Sargent, W. L. W. 1997, ApJS, 112, 315

Hopkins, P. F., Hayward, C. C., Narayanan, D., \& Hernquist, L. 2012, MNRAS, 420, 320

Hopkins, P. F., Hernquist, L., Cox, T. J., et al. 2006, ApJS, 163, 1

Hummel, E., van der Hulst, J. M., Keel, W. C., \& Kennicutt, R. C., Jr. 1987, A\&AS, 70, 517
Jaffe, W., Meisenheimer, K., Rttgering, H. J. A., et al. 2004, Natur, 429, 47 Kadler, M., Kerp, J., Ros, E., et al. 2004, A\&A, 420, 467

Maoz, D., Nagar, N. M., Falcke, H., \& Wilson, A. S. 2005, ApJ, 625, 699

Morganti, R., de Zeeuw, P. T., Oosterloo, T. A., et al. 2006, MNRAS, 371, 157

Müller-Sánchez, F., Davies, R. I., Eisenhauer, F., et al. 2006, A\&A, 454, 481

Müller-Sánchez, F., Davies, R. I., Genzel, R., et al. 2009, ApJ, 691, 749

Müller-Sánchez, F., Gonzlez-Martn, O., Fernndez-Ontiveros, J. A., AcostaPulido, J. A., \& Prieto, M. A. 2010, ApJ, 716, 1166

Müller-Sánchez, F., Prieto, M. A., Hicks, E. K. S., et al. 2011, ApJ, 739, 69

Nenkova, M., Ivezić, Z., \& Elitzur, M. 2002, ApJL, 570, 9

Nenkova, M., Sirocky, M. M., Nikutta, R., Ivezić, Ž., \& Elitzur, M. 2008, ApJ, 685,160

Orienti, M., \& Prieto, M. A. 2010, MNRAS, 401, 2599

Pogge, R. W., Maoz, D., Ho, L. C., \& Eracleous, M. 2000, ApJ, 532, 323

Prieto, M. A., Reunanen, J., Tristram, K. R. W., et al. 2010, MNRAS, 402, 724

Raluy, F., Planesas, P., \& Colina, L. 1998, A\&A, 335, 113

Sani, E., Davies, R. I., Sternberg, A., et al. 2012, MNRAS, 424, 1963

Schartmann, M., Meisenheimer, K., Camenzind, M., et al. 2008, A\&A, 482, 67

Schawinski, K., Urry, C. M., Virani, S., et al. 2010, ApJ, 711, 284

Tan, J. C., Beuther, H., Walter, F., \& Blackman, E. G. 2008, ApJ, 689, 775

Terashima, Y., \& Wilson, A. S. 2003, ApJ, 583, 145

Tristram, K. R. W., Meisenheimer, K., Jaffe, W., et al. 2007, A\&A, 474, 837

Tristram, K. R. W., \& Schartmann, M. 2011, A\&A, 531, 99

Vollmer, B., Beckert, T., \& Davies, R. I. 2008, A\&A, 491, 441

Wada, K., Papadopoulos, P. P., \& Spaans, M. 2009, ApJ, 702, 63

Wrobel, J. M., \& Heeschen, D. S. 1984, ApJ, 287, 41

Zhang, W. M., Soria, R., Zhang, S. N., Swartz, D. A., \& Liu, J. F. 2009, ApJ, 699,281 